\title{
Übersetzung und Mehrsprachigkeit im 12. Jahrhundert: Hermann von Karinthia
}

\section{Gerhard Katschnig}

Freiberuflicher Lektor und Kulturwissenschafter

\begin{abstract}
The period from the eighth to the twelfth century witnessed an unprecedented level of translation activity that changed the cultural and linguistic map. The flowering of knowledge in the fields of philosophy, astronomy, mathematics and astrology that started in the Islamic world later provided the impetus for the development of all branches of science in the Latin West. Hermann of Karinthia belonged to the first generation of European translators and is credited for the translation from Arabic into Latin of at least fifteen works-including Abū
\end{abstract} nisphaerium and his contribution to the first translation of the Qur'an under the guidance of Peter the Venerable. By examining Hermann's translations, this essay offers a contextualised reading of his works to see how revolutionary translation was rooted in the cultural-historical concerns of the twelfth century.

Keywords: Hermann of Karinthia, Peter the Venerable, Cultural History, Translational Turn, Cultural Transfer from Arabic to Latin Science, Late Middle Ages

(c) by the author; gerhard.katschnig@aau.at

Colloquium: New Philologies, Volume 5, Issue 2 (2020)

doi: 10.23963/cnp.2020.5.2.6

Stable URL: https://colloquium.aau.at/index.php/Colloquium/article/view/138

This work is licensed under a Creative Commons Attribution 4.0 International License (CC BY 4.0). 
Der folgende Beitrag widmet sich der Übersetzungsleistung des aus dem Alpen-AdriaRaum stammenden Philosophen, Mathematikers und Astronomen/Astrologen Hermann von Karinthia (um 1140). Zunächst wird eine Übersicht über den arabisch- und lateinischsprachigen Kulturtransfer antiken Wissens vom achten bis zum zwölften Jahrhundert geboten. Darauf aufbauend wird gezeigt, wie Hermanns vertiefende Auseinandersetzung mit dem arabischen Sprach- und Kulturraum zur Übertragung zahlreicher naturwissenschaftlicher und anderer Werke aus dem Arabischen ins Lateinische führte. Diese Rettung und Rückübersetzung eines Teils der antiken Werke, die Hermann und zahlreiche weitere Gelehrte zumeist in Arbeitsgruppen auf der Iberischen Halbinsel durchführten, gehört heute zu den Eckpfeilern europäischer Kultur- und Geistesgeschichte. ${ }^{1}$

\section{Antikes Wissen und Kulturtransfer}

\subsection{Antikes Wissen und Mönchskultur}

Einrichtungen in Mittel- und Westeuropa, die Wissen auf hohem Niveau vermittelten, gab es seit der Spätantike. Doch während im griechisch-orthodoxen Osten die bewährten Bildungsanstalten weiterhin Bestand hatten, wurde der lateinische Westen vom vierten bis zum sechsten Jahrhundert politisch instabil. Damit ging das vom dritten vorchristlichen Jahrtausend bis in die Zeit Mark Aurels (2. Jh.) entstandene Bildungsgut an Wissenschaften und Künsten zu einem großen Teil verloren und geriet zunehmend in Vergessenheit. Mit dem Zerfall des Imperium Romanum lösten sich die römischen Verwaltungsstrukturen und mit ihnen die öffentlich verwalteten Schulen auf, wodurch jegliche Wissensvermittlung in den privaten Bereich verlagert wurde. Mit der Ausbreitung des noch jungen Christentums übernahmen die entstehenden Klosterschulen die Aufgabe des gelehrten, auf Schriftlichkeit basierenden Unterrichts, während heidnische Sagen und Dichtungen in volkssprachlichen Überlieferungen lediglich weitgehend mündlich tradiert wurden (vgl. Nonn 2012, 10-14; Koch 2008, 68-71). Im zweiten Buch von De doctrina christiana, das in vier Bücher gegliederte Standardwerk zur christlichen Lehre, formulierte der bedeutende Kirchenlehrer Augustinus von Hippo (354-430) das für die folgenden Jahrhunderte entscheidende Bildungsprogramm:

Wenn aber diejenigen, die Philosophen genannt werden, zufällig etwas Wahres und zu unserem Glauben Passendes gesagt haben, wie besonders die Platoniker, dann darf dies nicht nur nicht gefürchtet, sondern muß sogar von diesen wie von ungerechten Besitzern für unseren Gebrauch eingefordert werden. [...] So bestehen alle Wissenschaften der Heiden nicht nur aus vor-

1 Für anregende Gespräche und Kritik danke ich Harald Krahwinkler und Mario Rausch. 
getäuschten und abergläubischen Erdichtungen und schweren Bürden von überflüssiger Mühe, [...] sondern sie enthalten auch die sogenannten freien Künste, die für den Nutzen der Wahrheit recht geeignet sind, und einige sehr nützliche Vorschriften zur praktischen Lebensführung. Selbst über die Verehrung des einzigen Gottes findet sich bei ihnen einiges Wahre. (Augustinus [Übers.], 97)

In der ausgewählten Passage argumentierte Augustinus für die Verbindung der antiken - und damit heidnischen - Wissenschaften und Künste mit dem christlichen Glauben. Diese Aneignung antiker Wissensbestände erfolgte über das von der römischen Kultur übernommene Bildungskonzept der sieben freien Künste (septem artes liberales), das für alle späteren Schulen des lateinischen Westens maßgeblich wurde. Reformiert wurde dieser Umstand durch das Bildungsbestreben Karls des Großen (747/48-814), die Geheimnisse des Glaubens in Psalmen und liturgischen Texten auf Latein subtiler zu vermitteln, als dies ein fränkischer Dialekt leisten konnte und bisher geleistet hatte. Mit der Bestellung Alkuins von York (ca. 735-804) als Berater an Karls Hof in der Königspfalz zu Aachen folgten entscheidende Impulse für die karolingische Bildungsreform. Alkuin entstammte der Tradition insularen Mönchtums, die einen größeren Wert auf Bildung und Didaktik der lateinischen Sprache legte als die meisten Klöster auf dem Festland. Er übernahm die Leitung der Hofschule, an der sich im Laufe der Jahre mehrere ,internationale“ Gelehrte versammelten, welche zur Grundlegung der geistigen Bildung in Europa ansetzten: Lehrwerke wurden verfasst, die im Karolingerreich als verbindliche Muster gelten sollten, und Bildungsbestrebungen - Verbesserung der Lateinkenntnisse und des Kirchenrechts, Aneignung antiker Bildungsinhalte für die kirchliche Bildung, Normierung der religiösen Praxis und Pflege der Volkssprachen - wurden an alle Reichsklöster sowie größeren Kirchen weitergegeben (Einhard 2006 [Übers.], cc. 25f.; Haberl 2014, 121-124; Kerner 2018, 287-290; Gleba 2004, 44ff.).

In den vielgestaltigen Dom- und Kathedralschulen, die wie in Paris oder Chartres zu Zentren höherer Bildung aufstiegen, wurden die freien Künste in Verbindung mit einem Verständnis ausgewählter biblischer Schriften und liturgischer Texte in lateinischer Sprache unterrichtet. Anders als im byzantinischen Osten, wo in den Sprachfächern des Triviums (Grammatik, Dialektik, Rhetorik) sowohl auf lateinische als auch auf griechische Texte zurückgegriffen wurde, blieb das professionelle Lesen und Schreiben auf die lateinische Sprache ausgerichtet - diese wurde bis zur Entstehung der ersten Universitäten und wohl teilweise darüber hinaus ausschließlich von Geistlichen vermittelt, welche die Spezialisierung des Wissens als klerikalen Bildungsmarker fest in ihrer Hand hielten. Sie konnten eine Expertenkultur etablieren, die sich durch das charakteristische Prinzip der Schrifthermeneutik gegenüber einer illiteraten und/oder paganen Volkskultur absetzte 
(vgl. Böhme 2011, 9). Damit vermittelten das lateinische Christentum und die sich konstituierende römische Kirche ein holistisches Kulturverständnis, das alle Möglichkeiten sowie Grenzen des Bildungserwerbs dominierte. Zugleich ist das Mittelalter nicht die Zeit, ,in der Europa christlich war, sondern in der es christlich wurde“ (Brunner 2012, 139). Im Zuge dieses Jahrhunderte währenden Prozesses geriet die Bezeichnung christlich nach römisch-lateinischer Normierung zu einem Kampf- und Ausgrenzungsbegriff gegen andere Glaubensvorstellungen, die entweder aus der Antike übernommen worden oder als Naturreligion vielfältig präsent waren, gegen expandierende arabisch-islamische Kulturen sowie gegen jene, deren Anspruch auf die Repräsentation des wahren Christentums andere Formen religiöser und sozialer Institutionen hervorgebracht hatte (vgl. Jussen 2016, 25ff.).

\subsection{Antikes Wissen und arabisch-islamischer Kulturtransfer}

Im Verlauf von Spätantike und frühem Mittelalter gab es nur wenige Gelehrte, die einen Kulturtransfer zwischen griechischem und lateinischem Wissen anstrebten. An dieser Stelle kann etwa Boethius angeführt werden, dessen Teilübersetzung von Euklids Elementen und des Gesamtwerks des Aristoteles zur Grundlage für den Logikunterricht und die Lehre der Trivium-Fächer der folgenden Jahrhunderte wurde (vgl. Vogel 2016, 13). Mit einem solchen Kulturtransfer werden im Anschluss an Hans-Jürgen Lüsebrink Prozesse der Übertragung und Vermittlung kultureller Artefakte - hierbei: Texte und Diskurse - anhand der Komponenten Selektion, Vermittlung und Rezeption bezeichnet. Dabei spielte es eine Rolle, welche Werke eines Sprach- oder Kulturraums mit den damit verbundenen Normen und Werten ausgewählt sowie übersetzt wurden, wer auf individueller, professioneller und institutioneller Ebene als Vermittlerinstanz fungierte sowie in welchen unterschiedlichen interkulturellen Aneignungsformen die Rezeption verlief (vgl. Lüsebrink 2016, 145-148). Der Selektionsprozess wurde im lateinischen Westen durch den Mangel an theologischen und philosophischen Schriften bestimmt, da diese in den Bibliotheken sowie Skriptorien rar waren und kaum rezipiert wurden. Waren vergleichbare antike Quellen vorhanden, trug der Rezeptionsprozess zur Wahrung des klerikal tradierten Bildungsgutes bei - vor allem Schriften von sowie Kommentare zu Aristoteles und Platon, die den Wert der kontrollierten sprachlichen Formulierung von These, Argument und Schlussfolgerung als Grundvoraussetzung für jegliche Form von wissenschaftlicher Erkenntnis vorgaben.

Dagegen konnte der arabisch-islamische Kulturraum den Reichtum antiker Wissenschaft und Philosophie durch eine frühe Rezeption transferieren und seit dem 7. Jahrhundert auf Bewahrung und Weiterentwicklung verweisen (vgl. Hunke 2005, 192; Bauer 2018, 75-83). Hier wurde der Selektionsprozess durch die Suche nach arabischen Ori- 
ginaltexten, arabischen Übertragungen griechischer, persischer und indischer Texte sowie nach griechischen, persischen und indischen Originaltexten aus der (Spät-)Antike bestimmt. In Bag̀dād, al-Bașra oder Ḥarrān gehörte das Studium griechischer Philosophie bereits im 8. Jahrhundert zum Kanon der Bildung, da griechische Werke wie jene des Aristoteles durch Übersetzungen ins Syrische in christlichen Städten wie Antiochia oder Edessa in die arabische Welt gelangt waren (vgl. Höffe 2014, 280f.). Unter den Abbasiden-Kalifen Hārūn ar-Raschīd (ca. 763-809) und seinem Nachfolger al-Ma'mūn (ca. 786-833) wurden vergleichbare Bildungsbestrebungen institutionalisiert, indem Generationen von griechisch- und syrischsprachigen Christen sowie jüdischen und arabischislamischen Wissenschaftern Übersetzungsaktivitäten forcierten. Das Herausragende bestand in der Vielfalt der Sprachen und der Wissensbereiche sowie im hohen Organisationsgrad der Mittlerinstitutionen. Erstmals wurden systematisch organisierte Übersetzungsprozesse initiiert, um Manuskripte aus dem griechischen, persischen und indischen Kulturraum, welche die Medizin, Astronomie und Mathematik, Politik und Philosophie behandelten, ins Arabische zu übertragen. Während Vermittlerkreis und Rezeption im lateinischen Westen durch die Klerikalisierung des Bildungswesens definiert wurden, waren es im arabischen Osten neben den Kalifen und Theologen auch Mediziner, Hofbeamte und Militärbefehlshaber, die als Übersetzer auftraten oder die institutionellen Bedingungen dafür schufen (vgl. Hourani 1992, 107ff.; Baker/Hanna 2011, 330; Sturlese 2013, 34).

Vergleichbar mit Karls Hof in Aachen wurde in Bag̀dād mit dem Haus der Weisheit ein Versammlungsort geschaffen, an dem sich ,internationale“ Gelehrte trafen, um eine Integration und dynamische Aneignung von Texten und Diskursen zur Theologie, Astronomie etc. zu ermöglichen sowie eine konzentrierte Übersetzertätigkeit im Umfeld der großen Palastbibliothek zu fördern. So kamen unter anderem der iranische Mathematiker und Geograf al-Hwwārizmī (ca. 780-835/850), der persische Astrologe und Mathematiker Abū Mǎšar (787-886) sowie der jüdische Philosoph und Astrologe Sahl ibn Bishr (9. Jh.) nach Bag̀dād, um hier zu studieren, eigenständige Werke zu schreiben und fremde Texte sowie Textkorpora zu übersetzen (vgl. al-Khalili 2016, 123-139). Bağdād übernahm für zwei Jahrhunderte die Rolle des Perikleischen Athen als gesellschaftspolitisch und kulturell höchst einflussreicher Ballungsraum in der arabischen Welt. Bis ins ausgehende 10. Jahrhundert wurden damit die bekanntesten Werke mehrfach ins Arabische übersetzt, studiert und kommentiert. Als Bagdād im Laufe des 11. Jahrhunderts von den Seldschuken erobert wurde, kamen manche Übersetzer und gründliche Kenner des Aristoteles und anderer Gelehrter nach Süditalien sowie auf die Iberische Halbinsel. ${ }^{2}$

2 Für die Übersetzertätigkeiten in Süditalien siehe Gázquez 2020, 99-110 und Freely 2019, 161-182. 
Während sich das arabische Zentrum von Wissenschaft und Bildung in der Folge nach Kairo und Damaskus verschob, setzte in Europa nach Augustinus die zweite Phase der Verbindung von christlicher Religion und antikem Wissen ein (vgl. Vietta 2007, 263). Wenn in diesem Zusammenhang von Bildungstradition oder der kulturellen Identität Europas gesprochen wird, sollte Folgendes, das selbst in neueren Publikationen noch ausgespart wird (vgl. Fuhrmann 2006) beachtet werden. Durch Nebenüberlieferungen oder (Rück-) Übersetzungen aus der arabischen Sprache erschloss sich der lateinischen Kulturwelt binnen weniger Jahrzehnte die erweiterte und revidierte Grundlegung europäischer Wissenschaft in Philosophie, Theologie, Jurisprudenz und Medizin.

\subsection{Die Übersetzerschulen in al-Andalus}

Im Laufe des 8./9. Jahrhunderts erreichten die arabisch-islamischen Expansionsbestrebungen weite Gebiete Süd- und Westeuropas. Als Teile der Iberischen Halbinsel (alAndalus) eingenommen wurden, begann ein kultureller Einfluss zu wirken, im Zuge dessen die arabische Wissenschaft und vor allem die arabischsprachigen Übertragungen ausgewählter griechischer Werke nach Europa gebracht wurden. Im Gegensatz zu Baġdād und anderen kulturellen Ballungszentren im Nahen Osten kam es in Córdoba, Toledo und weiteren Städten zu keiner markanten Übersetzungstätigkeit, da die benötigten Bücher bereits ins Arabische übertragen worden waren. Andalusische Herrscher wie al-Hakam II. (915-976), der Kalif von Córdoba, waren hingegen bemüht, die kulturpolitische Vormachtstellung zu festigen. Sie errichteten Moschen, förderten Künste sowie Gelehrtenkreise und bauten die eigenen Bibliotheken aus, indem arabischsprachige Werke aus den islamischen Reichen gesammelt und/oder kopiert wurden - unter anderem Texte von Klaudios Ptolemaios (ca. 100-160), die von dem in Madrid geborenen Astronomen Maslama al-Madschriti übertragen wurden (vgl. Szpiech 2019, 40f.; Glick 2005, 302). Mit dem Niedergang des Kalifats zerfiel al-Andalus in mehrere Stadtstaaten, was die (Rück-)Eroberung ehemals christlichen Herrschaftsgebietes begünstigte. Die sogenannte Reconquista gewann im 11. Jahrhundert an Intensität, als die bedeutendsten hispanischen Herrscherfamilien den römischen Ritus annahmen, um damit die römische Kirche zur Unterstützung zu gewinnen. Das kreuzzugsähnliche Gebaren, das aus einer Mischung aus politischen Überlegungen, Hoffnung auf Landgewinn und feudalen Lehensbindungen bestand, erreichte in der ersten Hälfte des 12. Jahrhunderts mit der Einnahme Zaragozas und Córdobas seinen vorläufigen Höhepunkt (vgl. Jaspert 2013, 110-114).

Der seit Ende des 11. Jahrhunderts auch infolge der Kreuzzüge zunehmende Kontakt mit dem Islam, der auf der Iberischen Halbinsel eine institutionell stark verankerte Traditionsgemeinschaft darstellte, führte zu einem Wechselspiel von kultureller und re- 
ligiöser Grenzziehung, das mit Feuer und Schwert nicht zu gewinnen war (vgl. Tischler 2011b, 169). Als theologische Eiferer im Hochmittelalter zu den Kreuzzügen aufriefen, erlebten und lebten Teile Spaniens die knapp zwei Jahrhunderte andauernde, auf Mehrsprachigkeit aufbauende, nicht konfliktfreie, aber kooperative Zeit der convivencia aller drei Abrahamitischen Religionen (vgl. Geier 2012, 40-44). In Toledo, Córdoba, Tudela und weiteren Gebieten des Iberischen Kulturraums fanden interreligiöse und interkulturelle Begegnungen statt, die eine beispiellose Integration griechischer, arabischer und jüdischer Kultur sowie Wissenschaft in das lateinisch-christliche Weltbild bedingten. In jedem dieser Bildungszentren fanden sich Mäzene, die auswärtige Gelehrte einluden und Übersetzungen von medizinischen, astronomischen/astrologischen, juristischen sowie naturwissenschaftlichen Werken in Auftrag gaben. Wenn wir an den vorher skizzierten Kulturtransfer anschließen, so wird im Sinne des translational turn in der Kulturwissenschaft im Folgenden eine Übersetzung von und zwischen den Kulturen ersichtlich, die nicht bei der Wort-für-Wort-Übertragung in eine andere Sprache mit korrekter Syntax und Grammatik stehen bleibt. Im Gegensatz zur lateinischen Mönchskultur, welche die Rezeption des antiken Wissens in den Dienst der Bibelexegese stellte, forcierten die Übersetzerschulen die Übertragung außereuropäischer Schlüsseltexte mit ihren disparaten Bedeutungsstrukturen in europäische Textformen. An die Stelle einer textfixierten Literaturübersetzung trat ein dialogischer und interaktionsbetonter Übersetzungsprozess, der zum Medium der Repräsentation fremder Kulturen und Wissensbestände wurde. Das Hin-und-her-Übersetzen von unterschiedlichen sprachlichen Zugehörigkeiten und die wechselseitigen Übersetzungspraktiken sollten die Übertragung unbekannter Denkweisen und Weltbilder sicherstellen. Zugleich darf nicht ausgespart werden, was bei jenen Manuskripten deutlich wurde, welche den Islam und den Propheten Muhammad betrafen: Übersetzungen von fremden Religionsvorstellungen stellten ein kulturpolitisches Projekt dar, das Ausdruck von Machtbeziehungen und Verzerrungen sein konnte (vgl. Bachmann-Medick 2010, 238-255; Bachmann-Medick 2011, 449-456).

Toledo stand in kirchenpolitischer Hinsicht seit dem 11. Jahrhundert unter dem Einfluss der Cluniazenser-Mönche. Diese schickten im Rahmen ihrer von Cluny ausgehenden monastischen Reformbewegung Emissäre nach Spanien und gründeten dort eine Vielzahl an Tochterklöstern. Nach Toledo kam Raymond de Sauvetât, der 1125 zum Erzbischof gewählt wurde (vgl. Schipperges 1957, 118f.; Henriet 2004, 69). Unter Raymonds Einfluss, der zugleich als Kanzler König Alfons ' VII. (1105-1157) agierte, wurde ein Gelehrtenzentrum begründet, das heute als Übersetzerschule von Toledo bekannt ist (vgl. Bossong 2010, 74-76). Gelehrte aus aller Welt wurden materiell bestens ausgestattet, um Werke aus der griechischen Antike sowie von arabischen Gelehrten aus dem 8. und 9. Jahrhundert zu übersetzen. Um ein optimales Verständnis des Ausgangstextes zu 
erreichen und um gleichzeitig gegen mangelnde Sprachkompetenz Qualitätssicherung zu betreiben, wurde vielfach in Zweierteams vorgegangen, die aus Einheimischen und $\mathrm{Zu}$ gereisten bestanden: Vermutlich übersetzten mozarabische oder jüdische Gelehrte, die Hocharabisch und/oder Griechisch beherrschten, zum Allgemeinverständnis mündlich in eine romanische Umgangssprache, ehe christliche Kleriker oder Gelehrte, die für ihre Übersetzungsarbeit als Domkapitulare angestellt wurden, diese mündliche Version lateinisch verschriftlichten (vgl. Burnett 1985, 166; König 2015, 474f.). Da die griechischen Originaltexte vielfach nicht vorhanden waren, handelte es sich bei den meisten Übertragungen um Übersetzungen von Übersetzungen. Neben natur- und rechtswissenschaftlichen Werken wurden ferner jene Schriften, welche die Glaubenslehre des Islam betrafen, für eine Übersetzung interessant. So beauftragte der Abt von Cluny, Petrus Venerabilis (ca. 1092-1156), Anfang der 1140er-Jahre den englischen Theologen und Astronomen Robert of Ketton (ca. 1110-1160), als erster Europäer den Koran ins Lateinische zu übersetzen.

\section{Hermann von Karinthia}

\subsection{Biografische Anmerkungen}

$\mathrm{Zu}$ Hermann von Karinthia gibt es bis dato keinen auf gesicherten Fakten basierenden, konsistenten Lebenslauf, da nicht genügend Primärquellen vorhanden sind, die verlässliche Rückschlüsse erlauben. Die wenigen Lebensstationen, die historisch als gesichert gelten, ermöglichen im Folgenden, biografische Anmerkungen wiederzugeben (vgl. PaićVukić 2019, 86-91; Kutleša 2004, 57f.; Burnett 1977a, 70). ${ }^{3}$ Demnach wuchs Hermann Anfang des 12. Jahrhunderts im Gebiet des Alpen-Adria-Raums irgendwo zwischen Istrien im Süden und dem österreichischen Bundesland Kärnten im Norden auf. ${ }^{4}$ Die vage Bestimmung seines Geburtsortes förderte in den letzten Jahrzehnten patriotische Motive der Zueignung: Hermann wird mit unterschiedlicher Deutungshoheit als herausragender Gelehrter slowenischer, kroatischer oder österreichischer Herkunft gesehen. Ebenfalls vage bestimmt bleibt sein Beiname: Neben Karinthia/Carinthia gelten die Varianten Secundus, Sclavus, Nellingaunensis und Dalmata, welche allesamt in unterschiedlichen Quellen die gleiche Person bezeichnen, um sie von Hermannus Contractus (10131054) oder Hermannus Alemannus (13. Jh.) zu unterscheiden.

\footnotetext{
3 Hervorzuheben ist in diesem Zusammenhang der historische Roman von Mario Rausch (Rausch 2020), der im Stil eines Itinerariums die wichtigsten Lebensstationen des hochmittelalterlichen Gelehrten erzählt.

4 Im Vorwort zur Übersetzung von Abū Ma šars Introductorium in Astronomiam heißt es: „,in medio patria nostra Karinthia“. Siehe: Hermann of Carinthia 1982, 346 und Haskins 1924, 54. Es wäre wenig gefehlt, Hermann in diesem Zusammenhang den unbestimmten Herkunftsnamen Undecumque zu geben.
} 
In seiner Jugend dürfte Hermann von Karinthia in einer benediktinischen Klosterschule mit dem klassischen Bildungskanon der freien Künste unterrichtet worden sein. Seine weiteren Stationen werden durch einen indirekten Verweis angedeutet, den der englische Kosmograf und Schriftsteller Richard Hakluyt (ca. 1552-1616) in seinem mehrbändigen Reisekompendium The Principal Navigations, Voyages, Traffiques, and Discoveries of the English Nation aus dem Eintrag eines anonymen Chronisten übersetzt wiedergab:

THis Robert Ketenensis was called an Englishman by surname, as he was by birth: who after some time spent in the foundations of humanitie, and in the elements of good Artes in the Vniuersities of England, determined to trauaile to the partes beyond sea: and so trauailed through France, Italie, Dalmatia, and Greece, and came at last into Asia, where he liued in great danger of his life among the cruell Saracens, but yet learned perfectly the Arabian tongue. Afterwardes he returned by sea into Spaine, and there about the riuer Iberus, gaue him selfe wholy to the studie of Astrologie, with one Hermannus a Dalmatian, who had accompanied him in his long voyage. He flourished in the yeere 1143. (Hakluyt 1888, 29f.)

Dem Bericht ist zu entnehmen, dass Hermann Robert of Ketton kennenlernte, mit dem ihn eine fortwährende Gemeinschaft und Zusammenarbeit verband. Die später mehrfachen Erwähnungen in ihren Werken zeugen von gegenseitiger Wertschätzung in der wissenschaftlichen Zusammenarbeit. Wenn wir die Aufzählung der Stationen chronologisch auffassen, so könnte Hermann Robert in Frankreich an der Domschule von Chartres oder in Paris erstmals begegnet sein, wo sie beide eventuell von Thierry de Chartres (ca. 1085-1150) und Bernardus Silvestris (ca. 1085-1160/1178) unterrichtet wurden. Zwei Hinweise für Hermanns Aufenthalt in Frankreich sprechen dafür: Zum einen widmete Hermann die Übertragung des Planisphaerium von Ptolemaios Thierry de Chartres, den er im Vorwort als seinen Lehrer und großen Kenner der lateinischen Studien bezeichnete (vgl. Lejbowicz 2003, 353). ${ }^{5}$ Der französische Philosoph förderte Übersetzungen aus dem Griechischen und Arabischen, wodurch bereits im frühen 12. Jahrhundert Manuskripte nach Chartres gebracht und dort übertragen wurden (vgl. Burnett 2013, 371). Zum anderen liefert ein Manuskript des englischen Chronisten und Historiografen Matthew of Paris (ca. 1200-1259), das mehrere Textsammlungen zur Wahrsagerei enthält, einen Hinweis, dass Hermann zu Bernardus Silvestris Kontakt hatte. Dem ersten Traktat,

5 Hermann of Carinthia 1982, 349: „[...] et fateor [...] tibi, inquam, diligentissime preceptor Theodorice, quem haut equidem ambigam, Platonis animam celitus iterum mortalibus accomodatam. [...] unum te Latini studii patrem $[\ldots]$..” 
dem Liber experimentarius, der Silvestris zugeschrieben wird, ist eine Abbildung vorangestellt, die Euklid und eine als Hermannus bezeichnete Person darstellt.

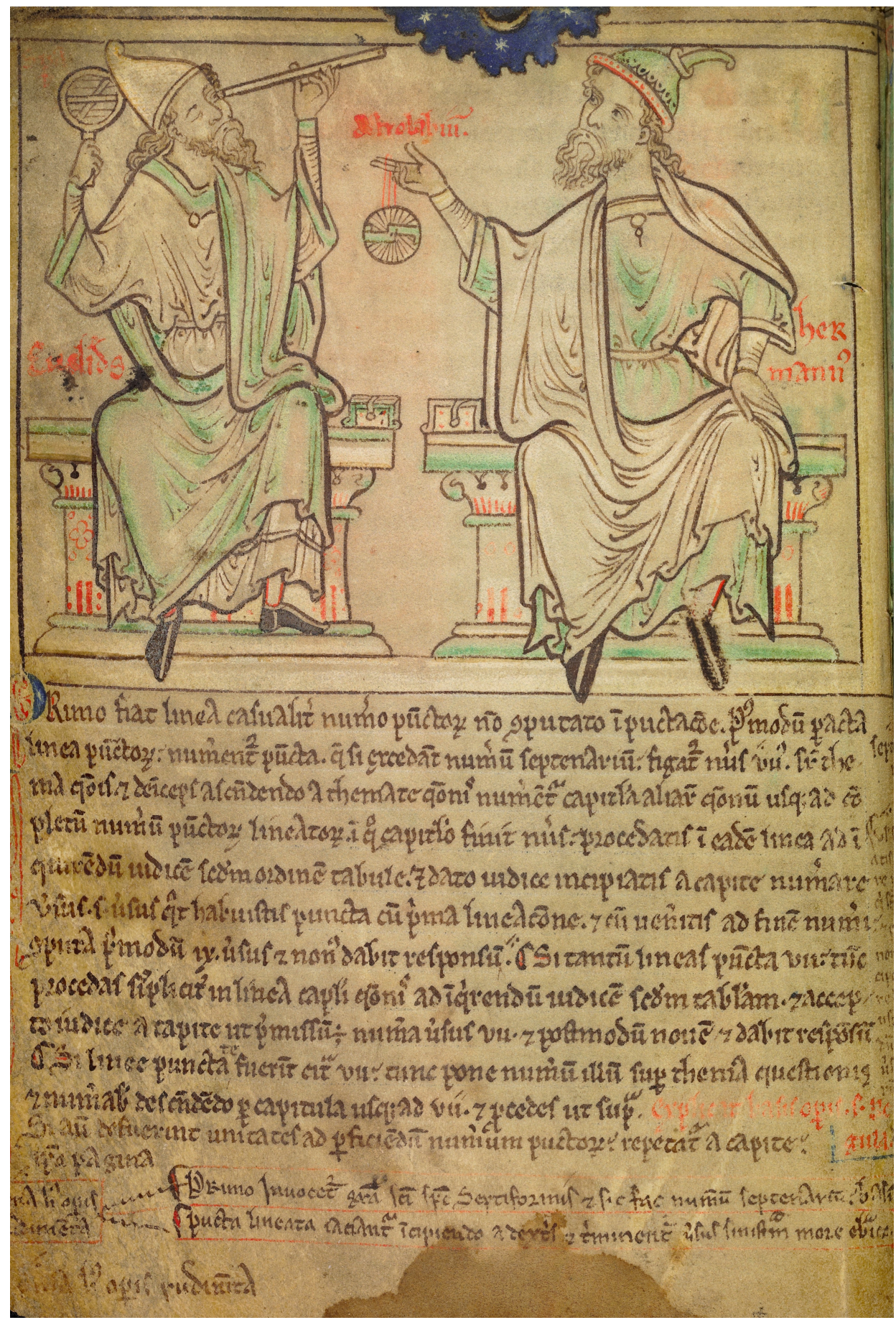

Abbildung 1: Euklid und Hermannus. In: Matthew of Paris: Texts on Prognostication ca. 1240 (Bodleian Library MS. Ashmole 304, fol. 2v). 
Es dürfte sich dabei um Hermann von Karinthia handeln, der Euklid ein Astrolabium mit Alhidade reicht, während dieser eine Armillarsphäre in seiner rechten Hand hält und mit der linken eine Visiereinrichtung adjustiert, um in die Sterne zu blicken. Als Kennzeichen für Autorität und Gelehrsamkeit, die aus einer anderen Zeit stammen, trägt Euklid im Gegensatz zu seinem Übersetzer Hermann eine phrygische Mütze. Nach dieser Lesart personifizieren die beiden Gelehrten Geometrie und Astrologie als jene wissenschaftlichen Disziplinen, die erforderlich waren, um den Himmel zu deuten und die Bewegung der Sterne zu erfassen (vgl. Burnett 1977b, 79; Iafrate 2013, 146-150). ${ }^{6}$

Nach weiteren, nicht näher definierten Aufenthalten in Italien, Dalmatien und Griechenland kamen Hermann und Robert dem Bericht nach in die Region Asia, die der Chronist mit dem Herrschaftsgebiet der Sarazenen gleichsetzte. Unter den Sarazenen dürfte er als diffuse Sammelbezeichnung islamisierte Völker im Nahen Osten subsumiert haben, bei denen Robert und Hermann mit arabischer Sprache, Kultur und vermutlich auch Wissenschaft vertiefend in Kontakt kamen. Ob sie dabei, wie manche Forscher der Biografie Hermanns zuschreiben, Damaskus, Konstantinopel, Bag̀dād, Kairo und/oder weitere Bildungszentren passierten, lässt sich nicht eruieren. Die Bildungsreise der jungen Wissenschafter als solche, die nicht erst eine Erfindung des 18. Jahrhunderts ist, erscheint realistisch, zumal die 1130er-Jahre frei von militärischen Unternehmungen im Rahmen der Kreuzzüge waren. Um 1138 kehrten sie nach Europa zurück und fuhren nach Nordspanien, wo sie in der Gegend um Tudela und Tarazona zusammen mit dem Theologen Hugo von Santalla und Hermanns Schüler Rudolph von Brügge - neben den bekannteren Bildungszentren - als eigenständige Gruppe begannen, arabische Manuskripte zu übersetzen (vgl. Lemay 1963, 644f.). In diese kurze, aber intensive Phase der Übersetzungsleistungen fällt die Bekanntschaft mit Petrus Venerabilis, den Hermann und Robert 1141/42 kennenlernten. Weitere Arbeitsorte Hermanns dürften Bildungszentren in León und Toledo gewesen sein. Während Robert nach seinem Wirken als Übersetzer seine kirchliche Karriere auf der Iberischen Halbinsel fortsetzte, kehrte Hermann nach (Süd-)Frankreich zurück, wo er 1142/43 in Toulouse und Béziers weitere Übersetzungen anfertigte und sein eigenständiges Hauptwerk De Essentiis abschloss. Danach verliert sich jegliche Spur zu Hermann von Karinthia.

${ }^{6}$ Es ist verschiedentlich - auch bei Burnett und Iafrate - darauf hingewiesen worden, dass es sich dabei um Hermannus Contractus handeln könnte, der neben kompilatorischen historiografischen Arbeiten auch Abhandlungen über das Astrolabium und die Geometrie veröffentlichte sowie mathematische und astronomische Werke aus dem Arabischen übertrug. Da er weder zu Vorhersagen noch zu Euklid Schriftliches hinterließ und kein Zeitgenosse von Bernardus Silvestris war, ist es wahrscheinlicher, in der Abbildung Hermann von Karinthia anzunehmen. 


\subsection{Hermanns Werk im Spiegel seiner Übersetzungspraxis}

Das Textkorpus seiner Übersetzungen (vgl. Björnbo 1903, 130-133; Burnett 1978, 100134; Dadić 1995, 40ff.) besteht aus naturwissenschaftlichen Schlüsseltexten, die zum Teil bis in die frühe Neuzeit als Standardwerke galten. Dabei handelt es sich vorwiegend um Werke zur Mathematik und Astrologie, in kleinerem Umfang um Übertragungen arabischer Texte ins Lateinische, die den Koran, den Islam und religiöse Schriften über Muhammad behandeln. Hermanns persönliche Anmerkungen zu den Themen und Werken, welche er bearbeitete, konnten somit lediglich im Vorwort bzw. in der Einleitung zur jeweiligen Übersetzung aufscheinen. Sein erstes Werk war Sahl ibn Bishrs umfangreiches sechstes Buch über Astrologie, Prophezeiung und den Einfluss der Gestirne auf Umwelt und Leben des Menschen, Fatidica, das er im Oktober 1138 übersetzte. Die ersten fünf waren bereits von Johann von Sevilla ins Lateinische übersetzt worden. 1140 übersetzte Hermann Abū Ma šars Einführung in die Astronomie (Introductorium in Astronomiam), die dieser 840 auf Basis der Werke des Aristoteles geschrieben hatte. Es folgten Euklids Elemente, das bis in die frühe Neuzeit benutzte Standardlehrbuch über Arithmetik und Geometrie, die Tripolites Sphaerica des Theodosius von Bithynien und al-Ḩwārizmīs astronomisches Lehrwerk $Z i j$ al-Sindhind mit Kalenderberechnungen. Am 1. Juni 1143 beendete er in Toulouse seine Übersetzung des Planisphaerium von Ptolemaios, das von Maslama al-Madschriti in Córdoba ins Arabische übertragen worden war.

Der griechische Mathematiker und Astronom Ptolemaios verfasste um 150 n. Chr. den Almagest $^{7}$, das in der Antike und für spätere Jahrhunderte bedeutendste Lehrwerk zur Astronomie. Während im Almagest eine mathematische Beschreibung der sich bewegenden Himmelskörper erfolgte, die zur Propagierung des geozentrischen Weltbildes führte, wandte sich Ptolemaios in Planisphaerium den Prinzipien der Konstruktion eines Astrolabiums zu, welche, wie Hermann im Vorwort erklärte, die Grundbedingung darstellen, um ein kompetenter Astronom zu werden (vgl. Gázquez 2019, 106f.). Dabei ging er zu den Anfängen zurück, die er in Mesopotamien vermutete und in Indien sowie später in Ägypten weitergeführt sah, ehe sie Ptolemaios aufgriff und systematisierte. Hermann ließ keinen Zweifel aufkommen, dass es sich hierbei um eine exakte Wissenschaft handelte, die mit mathematischer Berechnung und genauer Beobachtung vollzogen werde.

Am Ende des Vorworts folgt eine Widmung an Thierry de Chartres, die im vorigen Kapitel bereits erläutert wurde. Dabei eröffnete er einen aufschlussreichen Einblick in die Übersetzungspraxis und in das Umfeld der Übersetzer an sich. Obgleich er weder

7 Die mehrfachen Verweise auf den Almagest in Hermanns Hauptwerk De Essentiis lassen vermuten, dass er das Werk im griechischen Original kannte und Teile davon ins Lateinische übertrug, ehe das gesamte Werk von Gherardo da Cremona (1114-1187) in Toledo übersetzt wurde. Siehe Kalenić 1986, 41-44. 
Auftraggeber noch Übersetzer namentlich nennt, verwendet er knapp ein Viertel des Vorworts darauf, den von Missgunst und gegenseitiger Beargwöhnung geprägten Alltag seines eigenen „Berufsstandes“ zu schildern. ${ }^{8}$ Man bekommt den Eindruck, dass er von einer schwelenden Konkurrenzsituation der Übersetzer und Gelehrten schreibt, die wie sie etwa auch früher im Umfeld der karolingischen Bildungsbemühungen in Aachen unter den zugereisten Gelehrten bekannt war (vgl. Fried 2014, 288f.) - um Anerkennung sowie Förderung ihrer jeweils eigenen Forschung bemüht waren und zugleich nicht davor zurückschreckten, Karrierechancen zuungunsten anderer zu nutzen. Es scheint, als hätte es neben Reconquista und Kreuzzugsproblematik an sich selbst in den herausgehobenen Bildungszentren Berufskonflikte gegeben, die abseits der Übersetzungsleistungen den großen Stellenwert der sogenannten convivencia relativieren.

Aufgrund des deutlichen Überwiegens von naturwissenschaftlichen Werken dürfte Hermann erst auf ausdrückliche Nachfrage hin begonnen haben, Texte über den Islam und über Muhammad zu übersetzen (vgl. Tischler 2011a, 145-148; Steinschneider 1877, 227-234). 1141/42 kam Petrus Venerabilis auf die Iberische Halbinsel, um eine Wallfahrt nach Santiago de Compostela und eine Visitation der cluniazensischen Klöster zu unternehmen. Angesichts der grassierenden kriegerischen Auseinandersetzungen dürfte er den Entschluss gefasst haben, einen anderen Weg der Vermittlung zwischen Christentum und Islam anzustreben. So versammelte er im Kloster Santa María la Real de Nájera in der Nähe von Tudela gelehrte Übersetzer unterschiedlicher Provenienz, um ein Übersetzungsprojekt in Auftrag zu geben, das den Koran sowie ausgewählte Hadithe betraf. Diese Texte sollten ins Lateinische übertragen werden, um europäischen Lesern erstmals die Möglichkeit zu bieten, einen theologischen Dialog mit dem Islam zu führen, der auf Textkenntnis beruhte (vgl. Lukas 2018, 235; Vones 2006, 217-222; Le Goff 1986, 24f.; Bishko 1956, 166-168). Im Prolog seiner bezeichnenderweise Contra sectam Saraceno-

8 Hermann of Carinthia 1982, 349: „[...] quanta presumptione astronomie nomen usurpant, qui necdum principium eius viderint, que sine tribus premissis ita recte possibilis est, ut Ycarus volare potuit, nisi forte his, qui novo freti ingenio conversis discipline gradibus a fine incipiunt, - qui tamquam neglecto naturali gressu retrocedentes postpositis nimirum luminibus cecum carpant iter necesse est; tertio vero ut, quoniam tanti viri primarium hoc opus celestisque scientie quasi clavem quandam labor noster nunc tandem Latio confert, antequam in profanas insidiantium manus incideret, tua sanctissima constaret auctoritate. Quantam enim putas hominum partem hoc tempore superstitem, que propria contenta sorte non alieni cupiditate boni ferveat aut potius odio contabescat? Que passio maxime Latinitatis inopiam hucusquc fovit, necdum, licet pereunte materia, quiescens; quin me quoque, qui longe inter alios latere putabam, usque adeo sepius impellat, ut, tamquam cedens invidie, voto remisso, tanto labore, potius ad commune quodlibet vivendi negotium confugiam - cum presertim cunctis iam animi divitiis postpositis, nihil preter fortuitas opum sarcinas in pretio videam - nisi unum te virtutis exemplar haberem; quem nec labor vincit, nec delicie temperant, nec denique potentissima pervertit ambitio; [...]. 
rum $^{9}$ betitelten Streitschrift erhalten wir einen Einblick in das hehre Erkenntnisinteresse dieses Projekts:

Ob also nun die Irrlehre Muhammads mit dem häßlichen Namen der Häresie belegt oder als heidnisch geschmäht wird - man muß gegen sie vorgehen und gegen sie schreiben. Doch weil die Lateiner und erst recht unsere Zeitgenossen durch den Untergang des alten Eifers [...] nur noch die Sprache beherrschen, ,in der sie geboren sind“ [...] begab [ich] mich also zu Kennern jener arabischen Sprache [...]. Mit Geld und guten Worten brachte ich sie schließlich dazu, die Schriften über Abkunft, Leben und Lehre dieses verdammungswürdigen Menschen sowie das Gesetzbuch selbst, den Koran, aus dem Arabischen ins Lateinische zu übertragen. Und damit der Übersetzung eine strenge Glaubwürdigkeit nicht fehle, damit nichts versehentlich unserer Kenntnis vorenthalten werden könne, habe ich den christlichen Übersetzern auch einen Muslim beigegeben. [...] Robert von Ketton, Hermann von Dalmatien und Peter von Toledo, der Muslim hieß Muhammad. [...] Dieses wurde in dem Jahr vollendet, als ich nach Spanien kam und u. a. mit Alfons [...] eine Unterredung hatte. Das war im Jahre des Herrn $1141 .^{10}$

Wenn nach Walter Benjamin eine gelungene Übersetzung Lesern gilt, die das Original nicht verstehen, und über das bloß Vermittelnde hinausgeht, indem dieses in der Übersetzung eine spätere und umfassendere Entfaltung erfährt (vgl. Benjamin 1972, 9-11), ist die Mitteilung des Petrus Venerabilis in mehrfacher Hinsicht bemerkenswert: Der Abt übte Kritik an dem durchschnittlich gebildeten Leser, der durch die christliche Mönchskultur sozialisiert wurde und Griechisch weder sprach noch schrieb. Da kein Ensemble an Texten zum Islam verfügbar war, konnte die Instrumentalisierung von Übersetzung und Mehrsprachigkeit nicht deutlicher formuliert werden. Die Arbeitsgruppen wurden mit

9 Ursprünglich wollte Petrus den Kreuzzugsprediger Bernard de Clairvaux (ca. 1090-1153) überreden, eine schriftliche Widerlegung der Glaubensinhalte des Islam zu verfassen. Als dieser ablehnte, ging Petrus selbst daran, ein mehrbändig angelegtes Werk zu verfassen, das aber unvollständig blieb und heute unter dem Titel Contra sectam [sive haeresim] Saracenorum bekannt ist.

${ }^{10}$ Petrus Venerabilis 1985, 52-55. Contra sectam Saracenorum. Prologus, $\$ 17$ : „Sive ergo Mahumeticus error haeretico nomine deturpetur sive gentili aut pagano infametur, agendum contra eum est, scribendum est. Sed quia Latini et maxime moderni antiquo studio pereunte [...] non nisi linguam suam noverunt, ,in qua nati sunt ${ }^{\prime}[. .$.$] Contuli ergo me ad peritos linguae Arabicae, [...] Eis ad transferendum de lingua$ Arabica in Latinam perditi hominis originem, vitam, doctrinam legemque ipsam, quae Alkoran vocatur, tam prece quam pretio persuasi. Et ut translationi fides plenissima non deesset nec quicquam fraude aliqua nostrorum notitiae subtrahi posset, Christianis interpretibus etiam Saracenum adiunxi. [...] Robertus Ketenensis, Armannus Dalmata, Petrus Toletanus. Saraceni Mahumetus nomen erat. [...] Hoc anno illo factum est, quo Hispanias adii et cum domino Aldefonso [...] colloquium habui. Qui annus fuit ab incarnatione domini MCXLI ${ }^{\mathrm{US}}$ “ 
Geld angeworben und bestanden aus Einheimischen (Peter von Toledo und ein Übersetzer mit dem Namen Muhammad) sowie Auswärtigen (Robert of Ketton und Hermann von Karinthia). Dem Arbeitsteam wurde schließlich Petrus von Poitiers, der Notar und Sekretär des Abts, an die Seite gestellt. Zu fünft sollte die korrekte und redliche Übertragung ins Lateinische sichergestellt werden. Die konkrete Motivation des Übertragungsprozesses lag offensichtlich darin, die differenten Wertvorstellungen und Identifikationsmuster eines mit dem Christentum konkurrierenden Religionssystems besser kennenzulernen, um diese zu widerlegen und Muslime zum Übertritt ins Christentum zu bewegen (vgl. Albayrak 2000, 17f.). Zugleich bleibt zu vermuten, dass sich die Übersetzer, die weniger an einer Widerlegung des Islam, sondern stärker an naturwissenschaftlichen Werken interessiert waren, nicht über die Tragweite ihres Übersetzungsprozesses im Klaren gewesen sein dürften. Zu dem in Auftrag gegebenen Übersetzungsprojekt steuerte Hermann von Karinthia neben seiner Mitarbeit an der Koran-Übersetzung, die Robert of Ketton mit Lex Mahumet Pseudoprophetae (Gesetz des Pseudopropheten Muhammad) betitelte, De Generatione Mahumet und Doctrina Mahumet, welche beide noch 1142 übertragen wurden, bei: De Generatione Mahumet, das Hermann in León übersetzte, bildet eine Sammlung von jüdisch-islamischen Schöpfungslegenden, Prophetengeschichten und die Genealogie Muhammads - Doctrina Mahumet ist eine in Dialogform verfasste Bekehrungslegende dreier jüdischer Fragesteller, die von Muhammad überzeugt und bekehrt werden (vgl. d'Alverny 1947/48, 82-85; Kritzeck 1964, 84-96; Wüstenfeld 1877, 48-50). Die später als Collectio Toletana bezeichnete Zusammenstellung ausgewählter Texte zum Islam wurde erst 1543 von Theodor Bibliander (1505/06-1564) in Basel gedruckt. ${ }^{11}$

Zu Hermanns eigenen Abhandlungen zählen die Kompilation De Indagatione Cordis, welche sich an verschiedenen arabischen Vorbildern zur Wahrsagerei orientierte, sowie eine Sammlung von arabisch- und lateinischsprachigen Regeln mit zum Teil indischem Ursprung für Wettervorhersagen, die unter dem Titel Liber Imbrium an ein Laienpublikum adressiert war - beide Arbeiten behandeln die mannigfache Funktionalität astrologischer Beobachtungen (vgl. Low-Beer 1979, 89; Jenks 1983, 189). Die Arbeit an seinem

\footnotetext{
${ }^{11}$ Bibliander trat 1532 als Hebräisch- und Griechischspezialist die Nachfolge Ulrich Zwinglis (1484-1531) in Zürich an. 1543 konnte er die erste gedruckte Ausgabe einer Koran-Übersetzung nach Widerstand von Sebastian Münster und anderen, die entschieden gegen die Veröffentlichung eines blasphemischen Werkes, wie es der Koran für sie darstellte, aufgetreten waren, durchsetzen. Der dreibändige Druck enthielt eine Bestandsaufnahme seiner Zeit zur Auseinandersetzung zwischen Christentum und Islam: Band 1 enthielt die Collectio Toletana, deren Untertitel Quo velut authentico legum divinarum codice Agareni et Turcae, aliique Christo adversantes populi reguntur darauf anspielte, dass es sich beim Koran um ein Gesetz handle, dem sich jene, die sich Christus widersetzen, unterwerfen müssten. Die Bände 2 und 3 gaben von Ricoldo da Monte di Croce (ca. 1243-1320) bis Martin Luther (1483-1546) unterschiedliche Standund Streitpunkte wieder, die seit dem 13. Jahrhundert formuliert worden waren (vgl. Elmarsafy 2009, 3-6; Bevilacqua/Loop 2018, 150).
} 


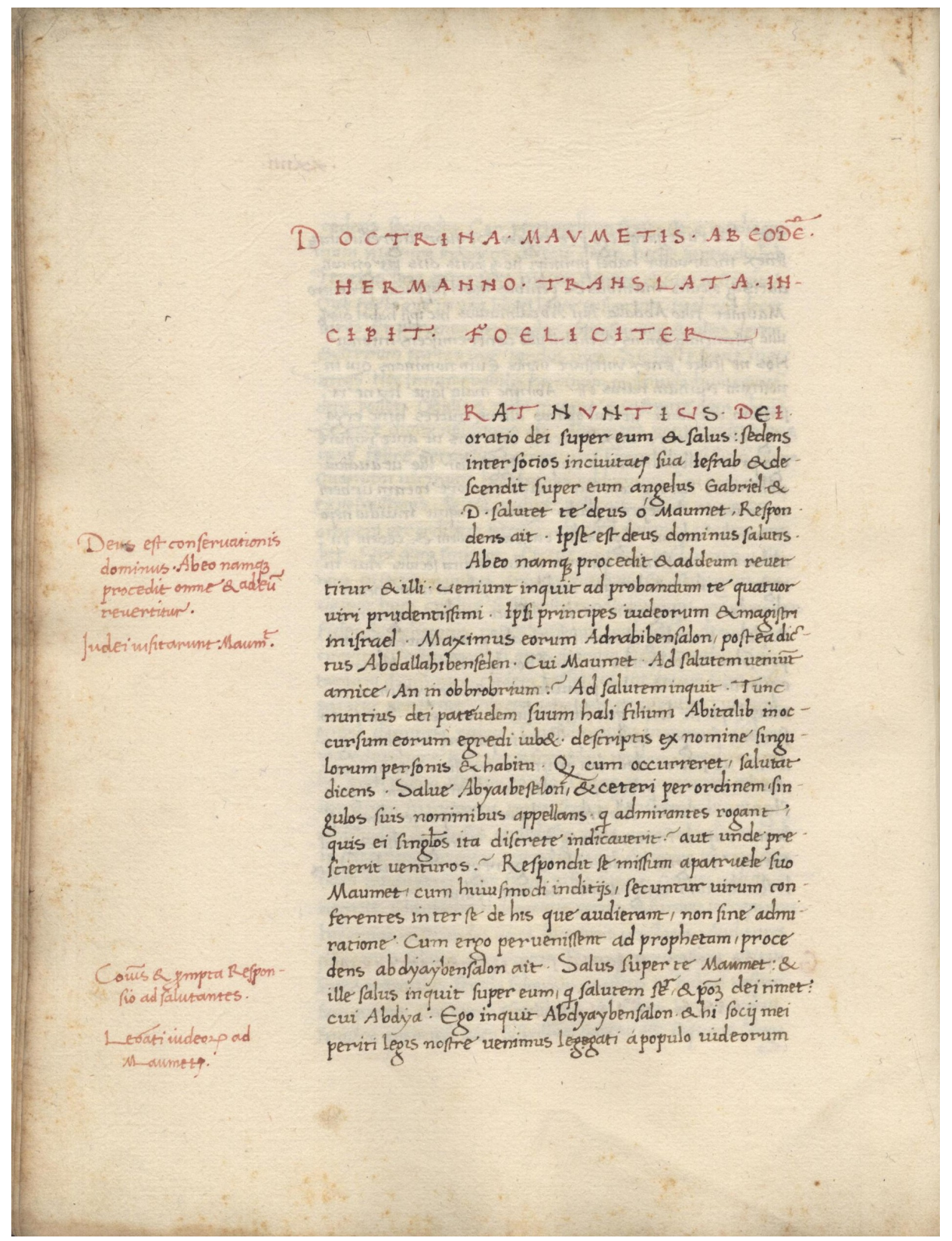

Abbildung 2: Doctrina Mahumet - ca. 16. Jahrhundert (SLUB, Ms. Dresd. A. 120.b, 24v).

Hauptwerk De Essentiis beendete er in der zweiten Jahreshälfte von 1143 in Béziers. Da keine späteren Arbeiten von ihm bekannt sind, stellt diese in zwei Büchern gegliederte Abhandlung seine abschließende Synthese von Astrologie und Kosmologie dar, die er auf Basis der aristotelischen Philosophie und unter Einbezug zusätzlicher antiker Quellen - von Platon, Boethius und weiteren - aus seinen bisherigen Übersetzungsarbeiten 
verfasste. Wenn es nach Robert of Ketton in einem Brief an Hermann, der dem ersten Buch vorangestellt wurde, außerhalb des wahren Glaubens an Gott keine Weisheit gäbe, ${ }^{12}$ so konnte Letzterer auf diese Einschätzung in zweierlei Hinsicht antworten: zum einen stimmte er zu, wenn wir die Erklärungsansätze theologischer Feinheiten zu Fragen der Trinität gegenüber den Überzeugungen islamischer Gelehrter wörtlich nehmen (vgl. Hermann of Carinthia 1982 [Übers.], 80ff., fol. 59r), zum anderen jedoch waren es die arabischen Schätze, die, wie Hermann von Karinthia im Proemium von De Essentiis erklärte, ihn öffentliche Feste ebenso wie andere Zweifel vergessen ließen. ${ }^{13}$

\section{Fazit}

In der Zeit vom achten bis zum zwölften Jahrhundert setzte ein bis dahin beispielloses $\mathrm{Maß}$ an Übersetzungsaktivität ein, das die kulturelle und sprachliche Landkarte Europas veränderte. Diese Expansion des Wissens in den Bereichen Philosophie, Astronomie, Mathematik und Astrologie begann in der arabisch-islamischen Welt und gab den Anstoß für die Entwicklung aller Wissenschaftszweige im lateinischen Westen. Hermann von Karinthia gehörte zur ersten Generation europäischer Übersetzer, die im Iberischen Kulturraum zahlreiche, vor allem die Naturwissenschaft betreffende Werke aus dem Arabischen ins Lateinische übertrugen. Diese Übersetzer und Kommentatoren waren kulturelle wie sprachliche Grenzgänger, die entweder in der mehrsprachigen andalusischen Gesellschaft aufgewachsen waren oder genügend Eifer zur Überschreitung ihrer eigenen Wissensbestände von auswärts mitbrachten. Einblicke in die Übersetzungspraktiken bei Hermann von Karinthia zeigten einerseits, dass auch sein Berufsstand nicht vor den Niederungen von Konkurrenz und Neid gefeit war, andererseits geriet die Arbeit an einer Übersetzung zum kulturpolitischen Instrument, wenn Religionsvorstellungen von und zwischen den Kulturen vermittelt werden sollten.

\section{Literatur}

Albayrak, Ismail. 2000. Qur'anic Narrative and Isra'iliyyat in Western Scholarship and in Classical Exegesis. Diss. Leeds.

al-Khalili, Jim. 2016. Im Haus der Weisheit. Die arabischen Wissenschaften als Fundament unserer Kultur. 2. Auflage. Aus dem Englischen von Sebastian Vogel. Frankfurt am Main: Fischer.

12 „extra veram divinitatis fidem nullum locum sapientie esse““ (Hermann of Carinthia 1982, 89, fol. 60r)

13 „Meministi, opinor, dum nos ex adytis nostris in publicam Minerve pompam prodeuntes, circumflua multitudo inhianter miraretur, non tanti personas pensans quantum cultus et ornatus spectans quos ex intimis Arabum thesauris diutine nobis vigilie laborque gravissimus acquisierat, [...].“ (Hermann of Carinthia 1982,70 , fol. $58 \mathrm{r}$ ) 
d'Alverny, Marie-Thérèse. 1947/48. „Deux Traductions Latines du Coran au Moyen Age." In Archives D'histoire Doctrinale et Littéraire du Moyen Age, 16: 69-131.

Augustinus, Aurelius. 2013. Die christliche Bildung (De doctrina christiana). Übersetzung, Anmerkungen und Nachwort von Karla Pollmann. Stuttgart: Reclam.

Bachmann-Medick, Doris. 2010. Cultural Turns. Neuorientierungen in den Kulturwissenschaften. Reinbek bei Hamburg: Rowohlt.

Bachmann-Medick, Doris. 2011. „Übersetzung als Medium interkultureller Kommunikation und Auseinandersetzung." In Handbuch der Kulturwissenschaften. Bd. 2: Paradigmen und Disziplinen, hrsg. von Friedrich Jaeger und Jürgen Straub, 449-466. Stuttgart/Weimar: Metzler.

Baker, Mona und Sameh Hanna. 2011. “Arabic Tradition.” In Routledge Encyclopedia of Translation Studies. Second Edition, edited by Mona Baker and Gabriela Saldanha, 328-337. New York: Routledge.

Bauer, Thomas. 2018. Warum es kein islamisches Mittelalter gab. Das Erbe der Antike und der Orient. München: Beck.

Benjamin, Walter. 1972. „Die Aufgabe des Übersetzers.“ In Ders. Gesammelte Schriften, Bd. IV/1, 9-21. Frankfurt am Main: Suhrkamp.

Bevilacqua, Alexander und Jan Loop. 2018. "The Qur'an in Comparison and the Birth of 'scriptures." In Journal of Qur'anic Studies, 20/3: 149-174.

Bishko, Charles Julian. 1956. “Peter the Venerable's Journey to Spain.” In Petrus Venerabilis 11561956: Studies and Texts Commemorating the Eighth Centenary of His Death, edited by Giles Constable and James Kritzeck, 163-174. (= Studia Anselmiana, 40). Rome: Herder.

Björnbo, Axel. 1903. „Hermannus Dalmata als Übersetzer astronomischer Arbeiten“. In Bibliotheca Mathematica. Zeitschrift für Geschichte der mathematischen Wissenschaften, 3/4: 130133.

Böhme, Hartmut. 2011. „Stufen der Reflexion: Die Kulturwissenschaften in der Kultur." In Handbuch der Kulturwissenschaften. Bd. 2: Paradigmen und Disziplinen, hrsg. von Friedrich Jaeger und Jürgen Straub, 1-15. Stuttgart: Metzler.

Bossong, Georg. 2010. Das Maurische Spanien. Geschichte und Kultur. 2. Auflage. München: Beck.

Brunner, Karl. 2012. Kleine Kulturgeschichte des Mittelalters. München: Beck.

Burnett, Charles. 1977a. "A Group of Arabic-Latin Translators Working in Northern Spain in the Mid-12th Century." In The Journal of the Royal Asiatic Society of Great Britain and Ireland, 1: 62-108.

Burnett, Charles. 1977b. "What is the 'Experimentarius' of Bernardus Silvestris? A Preliminary Survey of the Material." In Archives D'histoire Doctrinale et Littéraire du Moyen Age, 44: 79125 .

Burnett, Charles. 1978. "Arabic into Latin in Twelfth Century Spain: The Works of Hermann of Carinthia." In Mittellateinisches Jahrbuch, 13: 100-134.

Burnett, Charles. 1985. "Some Comments on the Translating of Works from Arabic into Latin in the Mid-Twelfth Century.” In Orientalische Kultur und europäisches Mittelalter, hrsg. von Albert Zimmermann und Ingrid Cremer-Ruegenberg, 161-171 (= Miscellanea mediaevalia, 17). Berlin/New York: de Gruyter, 1985. 
Burnett, Charles. 2013. “The Twelfth-Century Renaissance." In Cambridge History of Science, 2: 365-384, edited by David Lindberg and Michael Shanks. Cambridge: Cambridge University Press.

Dadić, Žarko. 1995. "Hermann the Dalmatian as Astronomer." In Hvar Observatory Bulletin, 19/1: 39-53.

Einhard. 2006. Vita Karoli Magni. Das Leben Karls des Großen. Übersetzung, Anmerkungen und Nachwort von Evelyn Scherabon Firchow. Stuttgart: Reclam.

Elmarsafy, Ziad. 2009. The Enlightenment Qur'an. The Politics of Translation and the Construction of Islam. Oxford: Oneworld Publications.

Freely, John. 2019. Platon in Bagdad. Wie das Wissen der Antike nach Europa kam. Aus dem Englischen von Ina Pfitzner. 6. Auflage. Stuttgart: Klett-Cotta.

Fried, Johannes. 2014. Karl der Große. Gewalt und Glaube. Eine Biographie. München: Beck.

Fuhrmann, Manfred 2006. Bildung. Europas kulturelle Identität. Stuttgart: Reclam.

Gázquez, José. 2020. “The Translations from Greek into Latin in the Middle Age.” In Revista Internacional d'Humanitats, 50: 99-110.

Gázquez, José. 2019. "The Importance of Ptolemy and the 'Almagest' in the Work of the Translators of Arabic Science in the Middle Ages." In Imago Temporis. Medium Aevum, 13: 97-113.

Geier, Wolfgang. 2012. Juden in Europa. Historische Skizzen aus zwei Jahrtausenden (= Wieser Enzyklopädie des europäischen Ostens, Bd. 9.1). Klagenfurt am Wörthersee: Wieser.

Gleba, Gudrun. 2004. Klosterleben im Mittelalter. Darmstadt: Wissenschaftliche Buchgesellschaft.

Glick, Thomas. 2005. Islamic and Christian Spain in the Early Middle Ages. Second, revised Edition. Leiden/Boston: Brill.

Haberl, Doris. 2014. „Die Hofbibliothek Karls des Großen als Kristallisationspunkt der karolingischen Renaissance: Geschichte, Umfeld, Wirkungen“. In Perspektive Bibliothek 3/1: 111139.

Hakluyt, Richard. 1888. The Principal Navigations, Voyages, Traffiques, and Discoveries of the English Nation. Vol. VIII: Asia. Part I, edited by Edmund Goldsmid. Edinburgh: Goldsmid.

Haskins, Charles. 1924. Studies in the History of Mediaeval Science. Cambridge: Harvard University Press.

Henriet, Patrick. 2004. "Un Bouleversement Culturel. Rôle et Sens de la Présence Cléricale Française dans la Péninsule Ibérique (xie-xiie siècles)." In Revue d'Histoire de l'Église de France, 90 (2004): 65-80.

Hermann of Carinthia. 1982. De Essentiis (= Studien und Texte zur Geistesgeschichte des Mittelalters, Bd. 15). A Critical Edition with Translation and Commentary by Charles Burnett. Leiden, Köln: E. J. Brill.

Hourani, Albert. 1992. Die Geschichte der arabischen Völker. Aus dem Englischen von Manfred Ohl und Hans Sartorius. Frankfurt am Main: Fischer.

Höffe, Otfried. 2014. Aristoteles. 4., überarbeitete Auflage. München: Beck.

Hoffmann, Andreas. 1829. „Herman der Dalmatier.” In Allgemeine Encyclopädie der Wissenschaften und Künste, 2/6: 258-259.

Hunke, Sigrid. 2005. Allabs Sonne über dem Abendland. Unser Arabisches Erbe. 5. Auflage. Frankfurt am Main: Fischer. 
Iafrate, Allegra. 2013. "Of Stars and Men: Matthew Paris and the Ilustrations of MS Ashmole 304." In Journal of the Warburg and Courtauld Institutes, 76: 139-177.

Jaspert, Nikolas. 2013. Die Kreuzzüge. 6., unveränderte Auflage. Darmstadt: WBG.

Jenks, Stuart. 1983. “Astrometeorology in the Middle Ages.” In Isis 74/2: 185-210.

Jussen, Bernhard. 2016. „,Abendland“ - ,Lateineuropa - ,Provincializing Europe‘: Bemerkungen zum poströmischen Europa zwischen alten und neuen Deutungsmustern." In Pluralistische Identität. Beobachtungen zu Herkunft und Zukunft Europas, hrsg. von Dirk Ansorge, 24-34. Darmstadt: WBG, 2016.

Kalenić, Antun. 1996. "Hermann of Dalmatia and Claudius Ptolemy." In Studia historiae philosophiae Croaticae, 3/3: 37-45.

Kerner, Max. 2018. „Alkuin und Karl der Große. Zur geistigen Grundlegung Europas. In Engagement und Diversität. Frank-Rutger Hausmann zum 75. Geburtstag, hrsg. von Wolfgang Asholt, Ursula Bähler, Bernhard Hurch, Henning Krauß und Kai Nonnenmacher, 285-296. Herausgegeben von. München: AVM.edition.

Koch, Hans-Albrecht. 2008. Die Universität. Geschichte einer europäischen Institution. Darmstadt: WBG.

König, Daniel. 2015. „Übersetzungskontrolle. Regulierung von Übersetzungsvorgängen im lateinisch/romanisch-arabischen Kontext (9.-15. Jahrhundert)." In Abrahams Erbe: Konkurrenz, Konflikt und Koexistenz der Religionen im europäischen Mittelalter, hrsg. von Klaus Oschema, 470-485. Berlin: De Gruyter.

Kritzeck, James. 1964. Peter the Venerable and Islam. Princeton: Princeton University Press.

Kutleša, Stipe. 2004. “Croatian Philosophers I: Hermann of Dalmatia (1110-1154).” In Prolegomena. Journal of Philosophy, 3/1: 57-71.

Le Goff, Jacques. 1986. Die Intellektuellen im Mittelalter. Aus dem Französischen übersetzt von Christiane Kayser. Stuttgart: Klett-Cotta.

Lejbowicz, Max. 2003. “Le premier témoin scolaire des 'Éléments' Arabo-latins d'Euclide: Thierry de Chartres et l“Heptateuchon”". In Revue D’histoire des Sciences, 56/2: 347-368.

Lemay, Richard. 1963. "Dans l'Espagne du XIIe siècle. Les Traductions de L'arabe au Latin." In Annales. Economies, Sociétés, Civilisations, 18/4: 639-665.

Low-Beer, Sheila. 1979. Hermann of Carinthia: The "Liber Imbrium", the "Fatidica", and the "De Indagatione cordis”. Diss. New York.

Lukas, Randolf. 2018. “'Non Armis Sed Verbis': Der lateinische christlich-islamische Dialog im Mittelalter und der Renaissance." In Forum Classicum, 4: 232-245.

Lüsebrink, Hans-Jürgen. 2016. Interkulturelle Kommunikation. Interaktion, Fremdwahrnehmung, Kulturtransfer. 4., aktualisierte und erweiterte Auflage. Stuttgart: Metzler.

Nonn, Ulrich. 2012. Mönche, Schreiber und Gelehrte. Bildung und Wissenschaft im Mittelalter. Darmstadt: Wissenschaftliche Buchgesellschaft.

Paić-Vukić, Tatjana. 2019. "The Biography of Hermann the Dalmatian: Separating Conjectures from Verifiable Claims." In Exploring the Commonalities of the Mediterranean Region: Proceedings of the TUBA-EMAN Symposium, hrsg. von Yurdusev Ahmet, 85-96. . Ankara.

Petrus, Venerabilis. 1985. Schriften zum Islam (= Corpus Islamo-Christianum. Series latina, Bd. 1).Ediert, ins Deutsche übersetzt und kommentiert von Reinhold Glei. Altenberge: CIS-Verlag. 
Rausch, Mario. 2020. Hermann de Carinthia. Eine Biographie. Klagenfurt am Wörthersee: Wieser Verlag.

Schipperges, Heinrich. 1957. „Das griechisch-arabische Erbe Toledos und sein Auftrag für die abendländische Heilkunde“. In Sudhoffs Archiv für Geschichte der Medizin und der Naturwissenschaften, 41/2: 113-142.

Steinschneider, Moritz. 1877. Polemische und apologetische Literatur in arabischer Sprache zwischen Muslimen, Juden und Christen, nebst Anhängen verwandten Inhalts. Leipzig: Brockhaus.

Sturlese, Loris. 2013. Die Philosophie im Mittelalter. Von Boethius bis Cusanus. München: Beck.

Szpiech, Ryan. 2019. "From Mesopotamia to Madrid: The Legacy of Ancient and Medieval Science in Early Modern Spain.” In Science on Stage in Early Modern Spain (= Toronto Iberic, 38), edited by García Santo-Tomás Enrique, 25-57. Toronto/Buffalo/London: University of Toronto Press.

Tischler, Matthias. 2011a. „Die Iberische Halbinsel als christlich-muslimischer Begegnungsraum im Spiegel von Transfer- und Transformationsprozessen des 12.-15. Jahrhunderts." In Anuario de Historia de la Iglesia, 20: 117-155.

Tischler, Matthias. 2011b. „Übersetzen als des/integrativer Akt. Die lateinischen Übertragungen arabischer muslimischer Literatur auf der Iberischen Halbinsel im 12. und 13. Jahrhundert." In Christlicher Norden - muslimischer Süden. Ansprüche und Wirklichkeiten von Christen, Juden und Muslimen auf der Iberischen Halbinsel im Hoch-und Spätmittelalter, hrsg. von Matthias Tischler und Alexander Fidora, 167-186. Münster: Aschendorff.

Vietta, Silvio. 2007. Europäische Kulturgeschichte. Eine Einführung. Paderborn: Fink Verlag. Vogel, Christian 2016. Boethius' Übersetzungsprojekt. Philosophische Grundlagen und didaktische Methoden eines spätantiken Wissenstransfers (= Episteme in Bewegung. Beiträge zu einer transdisziplinären Wissensgeschichte, Bd. 6). Wiesbaden: Harrassowitz.

Vones, Ludwig 2006. „Zwischen Kulturaustausch und religiöser Polemik. Von den Möglichkeiten und Grenzen christlich-muslimischer Verständigung zur Zeit des Petrus Venerabilis." In Wissen über Grenzen. Arabisches Wissen und lateinisches Mittelalter (= Miscellanea Mediaevalia, Bd. 33), hrsg. von Andreas Speer und Lydia Wegener, 217-237. Berlin/New York: de Gruyter.

Wüstenfeld, Ferdinand. 1877. Die Übersetzungen Arabischer Werke in das Lateinische seit dem XI. Jahrhundert. Aus dem zweiundzwanzigsten Bande der Abhandlungen der Königlichen Gesellschaft der Wissenschaften zu Göttingen. Göttingen: Dieterichsche Verlags-Buchhandlung. 